\title{
LINEAMIENTOS PEDAGÓGICOS PARA APLICAR TICS Y EVEA EN INSTITUCIONES EDUCATIVAS DE EDUCACIÓN BÁSICA Y BACHILLERATO EN GUAYAQUIL-ECUADOR ${ }^{1}$
}

\section{PEDAGOGICAL GUIDELINES FOR APPLYING ICT AND EVEA IN EDUCATIONAL INSTITUTIONS OF BASIC EDUCATION AND BACHELOR'S DEGREE IN GUAYAQUIL- ECUADOR}

Rosario Mercedes Peláez López, Mgs. ORCID 0000-0002-8320-0678 Magíster en Gerencia y liderazgo educacional (Ecuador). Rectora de la Unidad Educativa Particular “Americus Mundus NOVUS”. Directora Pedagógica de Jardineritos Pre-Escolar, Ecuador. rpelaez@centroeducativonovus.edu.ec

Jorge Adrian Alvarado Peláez, Ing. Ingeniero en Gestión Empresarial Internacional (Ecuador). Jefe de Marketing en Contífico, Ecuador. jalvarado@contifico.com

Ma. José Zambrano Peláez, Lcda. Licenciada en Párvulos (Ecuador). Directora General en la Unidad Educativa Particular “Americus Mundus NOVUS”, Ecuador. majozambranop78@gmail.com

${ }^{1}$ Artículo original derivado del proyecto de investigación titulado "El uso del EVEA y su relación con el Profesorado de la Unidad Educativa Steiner Internacional de la Sección Primaria, de la Ciudad de Guayaquil: un estudio de caso", financiado por Rosario Peláez López y realizado entre 2016 y 2018 


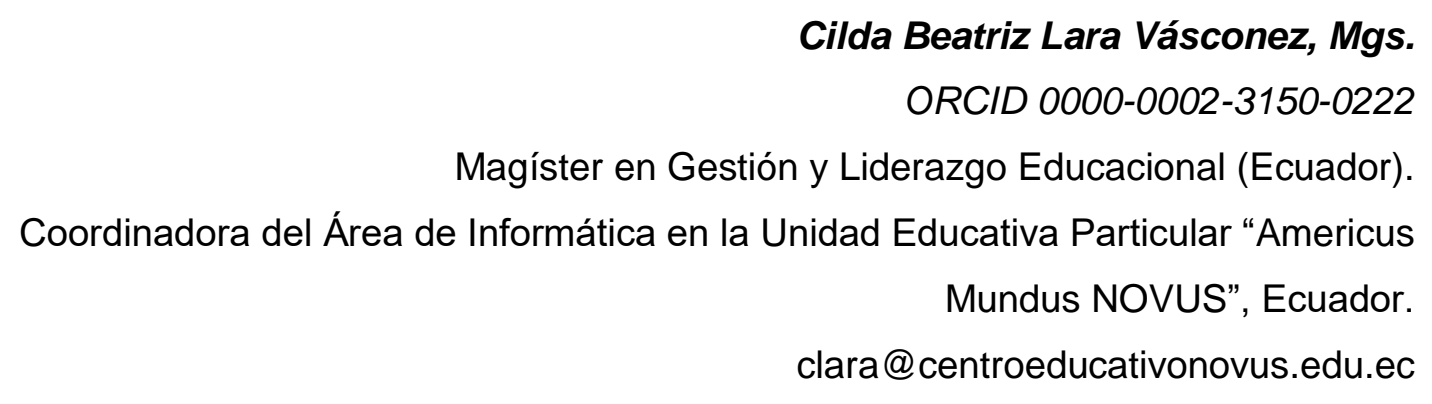

\section{ARTÍ́CULO DE INVESTIGACIÓN}

Recibido: 5 de noviembre de 2019.

Aceptado: 25 de diciembre de 2019.

\section{RESUMEN}

La tecnología con el paso del tiempo ha ido implementando diversas estrategias para el aprendizaje con la única intención de mejorar la calidad en la educación, motivo por lo cual en la actualidad se llega al educando desde Entornos Virtuales de Enseñanza-Aprendizaje (EVEA) muy amigables. A través del INEC se ha observado que el uso del internet alcanzó un total de $50,5 \%$ en el año 2015 y a nivel nacional, lo cual permite analizar la aplicabilidad en el uso de los EVEA en las instituciones educativas. El objetivo es propiciar la ayuda adecuada a todos los estudiantes en su educación utilizando dichos entornos virtuales, para así analizar el desempeño de los profesores con la utilización de las TIC promoviendo el uso de plataformas y el refuerzo de los contenidos en las instituciones educativas. La investigación es de tipo cuantitativa, se aplicaron encuestas, entrevistas y se realizó un estudio de campo para evidenciar la actuación y el desenvolvimiento de los docentes. Los resultados obtenidos después de la tabulación se orientan al avance tecnológico en el manejo por parte de algunos profesores, con la sugerencia de realizar capacitaciones constantes en el manejo de las TIC, además del uso de Programas como Presentadores Electrónicos.

Palabras clave: EVEA, TIC, aprendizaje, entornos virtuales. 


\section{ABSTRACT}

Technology over time has been implementing various learning strategies with the sole intention of improving the quality of education, which is why currently the student is reached from very friendly Virtual Teaching-Learning Environments (EVEA). Through INEC it has been observed that internet use reached a total of $50.5 \%$ in 2015 and at the national level, which allows us to analyze the applicability in the use of EVEAs in educational institutions. The objective is to provide adequate help to all students in their education using these virtual environments, in order to analyze the performance of teachers with the use of ICT, promoting the use of platforms and the reinforcement of content in educational institutions. The research is of a quantitative type, surveys, interviews were applied and a field study was carried out to demonstrate the performance and development of the teachers. The results obtained after the tabulation are oriented to the technological advance in the management by some teachers, with the suggestion to carry out constant training in the management of ICT, in addition to the use of Programs such as Electronic Presenters.

Keywords: EVEA, TIC, learning, virtual environments.

\section{INTRODUCCIÓN}

Dentro de las herramientas que las Tecnologías de la Información y Comunicación con que cuentan en los centros de educación están los Entornos Virtuales o Entornos Virtuales de Aprendizaje (Guaña - Moya, Llumiquinga-Quispe, \& Ortiz-Remache, 2015). Dichos entornos se definen como "un conjunto de facilidades informáticas y telemáticas para la comunicación y el intercambio de información en el que se desarrollan procesos de enseñanza-aprendizaje" (Gómez, Pérez, \& Tamayo, 2007), es decir, que propicia la adquisición de conocimiento mediante la enseñanza-aprendizaje convergiendo el trabajo docente ante la avidez del estudiante.

Con el desarrollo de tecnología surgen los Entornos personales de aprendizaje, los cuales permiten crear al estudiante su entorno para el aprendizaje debido a que dan lugar a personalizar herramientas que posibilitan la comunicación asincrónica con el tutor asignado en cada temática 
tratada (Ledo, Hernández, Cao, \& Vidal, 2015). En esa línea, existe software multimedia, Sistemas de Gestión de Aprendizaje, blogs, sitios web dedicados a la educación, plataforma Moodle y demás elementos, que pueden ser gratuitos o de pago, en línea o fuera de línea, etc. Muchos de ellos han servido de apoyo para la educación autónoma y como guía del docente.

El entorno virtual de enseñanza y aprendizaje (EVEA) es uno de los entornos que surgió en conjunto con las TIC en la educación, se considera un conjunto de herramientas informáticas y telemáticas que facilitan la comunicación e intercambio de información en el cual se desarrolla un proceso de enseñanza y aprendizaje. Los EVEA permiten la interacción de docentes, estudiantes, padres de familia, coordinadores educativos y autoridades, desde el punto de vista de la educación.

Rallo, et al. (1997) también define al EVEA "como el entorno adecuado para aplicar y reforzar técnicas del aprendizaje a distancia y aprendizaje cooperativo, usando de una forma eficiente todos los recursos tecnológicos disponibles". Al ser un instrumento de apoyo al proceso educativo y tener la facilidad de ser utilizado en cualquier lugar con acceso a internet, moviliza dicho proceso fuera del aula de clase, pero manteniendo la guía del docente. Es clave que el docente tenga conciencia de esta característica para que organice los elementos de tal manera que alcance el mayor provecho para el aprendizaje del estudiante, aunque no se encuentre físicamente.

Por otra parte, existe el uso de EVEA dentro de la institución educativa y en ese caso, en la planificación de la clase debe contemplarse momentos de quiebre donde el docente aporte a lo que se observa o explora en el entorno virtual. Lograr que la enseñanza del docente se alcance a través del vehículo del EVEA es consecuencia de una planeación que suponga escenarios de desatención del estudiante, de preguntas no contempladas, desmotivación, consideración de autosuficiencia del sistema, etc.

Además, es importante recalcar que los cambios a nivel de gobierno están promoviendo actualmente el lanzamiento de la Agenda Educativa Digital 2017-2021, cuyo objetivo es fortalecer y potenciar el proceso de enseñanza-aprendizaje en el Sistema Educativo Nacional a través del 
incremento de prácticas educativas que integren las tecnologías digitales para empoderar el aprendizaje, el conocimiento y la participación. Adicionalmente, sus pilares contribuyen a la mejora de la calidad educativa, la cobertura y la garantía de los derechos, la introducción de la innovación tecno-pedagógica en el Ecuador, con equipamiento, conectividad, formación y capacitación al profesor, la construcción de contenidos curriculares y recursos educativos; así como la ciudadanía digital para una buena convivencia en espacios virtuales y digitales, permitiendo generar estrategias de prevención en el buen uso de las tecnologías digitales y en los riesgos y peligros que se presentan en la navegación de Internet. (2019, p. 5).

Una ventaja en este tema es que en el Ecuador se han realizado varios estudios acerca del uso que los niños realizan del internet. El Instituto de Estadísticas y Censos (INEC) ha realizado varias indagaciones acerca de este aspecto; en el 2015, se observó un aumento promedio de $5 \%$ anual en el uso internet, alcanzando un $50,5 \%$ en dicho año a nivel nacional.

En la encuesta que se realizó en el año 2011 acerca del uso que le dan los niños al internet se pudo constatar que cada día el internet está más presente en los hogares ecuatorianos. De allí que los resultados fueron los siguientes:

Tabla 1. Uso de internet en los niños

\begin{tabular}{|l|c|c|}
\hline \multicolumn{3}{|c|}{ Uso que le dieron al Internet (Ios niños de 5 a 11 años en los últimos 12 meses) } \\
\hline & Niños & Niñas \\
\hline Educación y aprendizaje & $59,8 \%$ & $61,9 \%$ \\
\hline Obtener información & $18,9 \%$ & $19,9 \%$ \\
\hline Comunicación en general & $18,7 \%$ & $15,2 \%$ \\
\hline Actividades de entretenimiento & $1,4 \%$ & $1,3 \%$ \\
\hline Leer / descargar libros electrónicos & $0,3 \%$ & $0,5 \%$ \\
\hline Razones de trabajo & $0,1 \%$ & $0,1 \%$ \\
\hline
\end{tabular}

Fuente: Elaborado por Unidad de Procesamiento (UP) del INEC a partir de la Encuesta Nacional de Empleo, Desempleo y Subempleo (ENEMDU DIC- 2011). 
Los resultados muestran que los niños tienen interés en aprender a través del uso del internet, aunque no se especifica que el uso de EVEA sea un ambiente propicio para la educación y aprendizaje. Una vez más, puede indicarse que el docente es el principal impulsador para que un entorno virtual pueda ser utilizado para aprender por descubrimiento.

\section{MATERIALES Y MÉTODOS}

Durante esta investigación se realizó una exploración en tres unidades educativas del sector norte de la ciudad de Guayaquil, las cuales poseen los recursos e infraestructura necesaria para realizar uso de los TIC y EVEA sin limitaciones. Se aplicó encuestas a todos sus docentes de Educación General Básica llegando a una muestra de 80 participantes, quienes cumplen con el requerimiento de 40 años de edad como mínimo.

Tabla 2. Muestra de docentes por género y edades.

\begin{tabular}{|c|c|c|c|}
\hline \multicolumn{4}{|c|}{ Docentes por género y edades } \\
\hline \multicolumn{2}{|l|}{ Hombres } & \multicolumn{2}{|l|}{ Mujeres } \\
\hline $40-55$ años & $56-60$ años & $40-55$ años & $56-60$ años \\
\hline 22 & 8 & 44 & 6 \\
\hline Total & 30 & Total & 50 \\
\hline & & Total & 80 \\
\hline
\end{tabular}

Fuente: Elaboración propia.

Tabla 3. Muestra de docentes por años de experiencia.

\begin{tabular}{|l|l|l|l|}
\hline \multicolumn{3}{|c|}{ Docentes por años de experiencia } \\
\hline Hombres & Mujeres \\
\hline $5-15$ años & $16-30$ años & $5-15$ años & $16-30$ años \\
\hline
\end{tabular}

ISSN: 1390-9320, Edición Especial, febrero 2020 


\begin{tabular}{|l|l|l|l|}
\hline 15 & 15 & 15 & 35 \\
\hline Total & 30 & Total & 50 \\
\hline & & Total & 80 \\
\hline
\end{tabular}

Fuente: Elaboración propia.

Se estableció esta división de la muestra porque se asocia, parcialmente, tanto la edad del docente como sus años de experiencia a su aceptación de las nuevas tecnologías para aportar a su proceso de enseñanza. Como refleja la muestra, el $38 \%$ corresponde a hombres, de los cuales el $73 \%$ tiene entre $40-55$ años y el $27 \%$ de $56-60$ años. Las mujeres corresponden al $62 \%$ de la muestra, lo cual se considera una gran mayoría; el $88 \%$ tiene entre $40-55$ años y el $12 \%$ de $56-60$ años.

Este caso de estudio es cualitativo, en donde se detalla que es un estudio etnográfico y una investigación de acción en donde se destaca que la población que se ha elegido para la muestra es de 3 instituciones educativas que tienen características similares en cuanto al personal y recursos que poseen.

Los métodos utilizados para la recogida de datos que se aplicaron en esta investigación fueron: entrevistas, cuestionarios, observación y encuestas.

\section{RESULTADOS}

Los docentes fueron encuestados en el plantel donde laboran agrupando a todos los participantes. Al principio se brindó una introducción del tema a estudiar para exponer los objetivos y la normativa para completar el cuestionario. Se aseguró el anonimato tanto de los participantes como de las instituciones participantes en el presente estudio, motivando a la colaboración.

En el gráfico 1 se observan los resultados de uso de TIC en el proceso de enseñanza y aprendizaje de los docentes entre 40 a 55 años, donde se observa un 52\% empleando Siempre las TIC, el $32 \%$ Frecuentemente, el $13 \%$ A veces y el $3 \%$ Pocas veces. 


\section{PORCENTAJE}

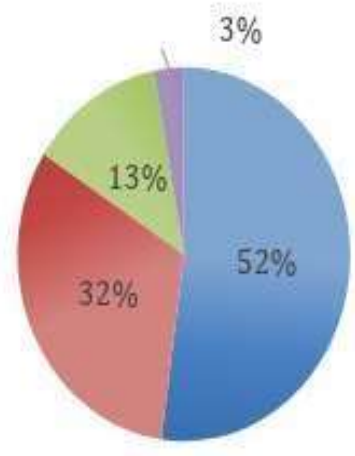

Siempre

Frecuentemente

A veces

Pocas veces

Nunca

Gráfico 1. Uso de las TIC en el proceso de enseñanza y aprendizaje (40-55 años)

Fuente: Elaborado propia.

En el gráfico 2 se encuentra reflejado el resultado de los docentes entre $56-60$ años, donde los resultados cambian y el $7 \%$ emplea Siempre las TIC, el $21 \%$ Frecuentemente y el $71 \%$ A veces.

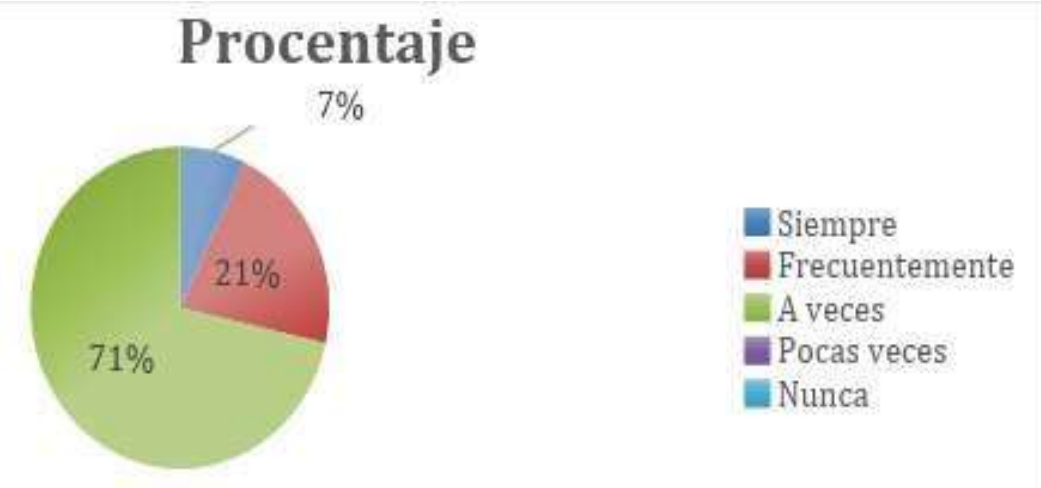

Gráfico 2. Uso de las TIC en el proceso de enseñanza y aprendizaje (56-70 años) Fuente: Elaboración propia.

Otra característica que resaltan en el uso de TIC y EVEA es los años de experiencia del docente, ya que, a partir de aquello, considera que sus métodos son los óptimos y existe resistencia al cambio. Es así que el gráfico 3, demuestra que los docentes que tienen entre 5 a 15 de años de experiencia, el $88 \%$ Siempre utilizan las TIC, el 6\% Frecuentemente y el 6\% A veces. 


\section{PORCENTAJE}

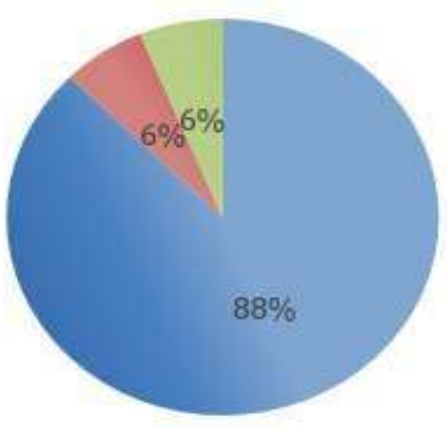

Siempre

Frecuentemente

A veces

Pocas veces

Nunca

Gráfico 3. Uso de las TIC en el proceso de enseñanza y aprendizaje por su experiencia (5-15 años)

Fuente: Elaboración propia.

Los docentes entre 16 - 30 años de experiencia constituyen la mayoría en esta categoría y responden de manera distinta respecto del uso de TIC. El 31\% las emplea Siempre, el 20\% Frecuentemente, el $45 \%$ A veces y el $4 \%$ Pocas veces. 6

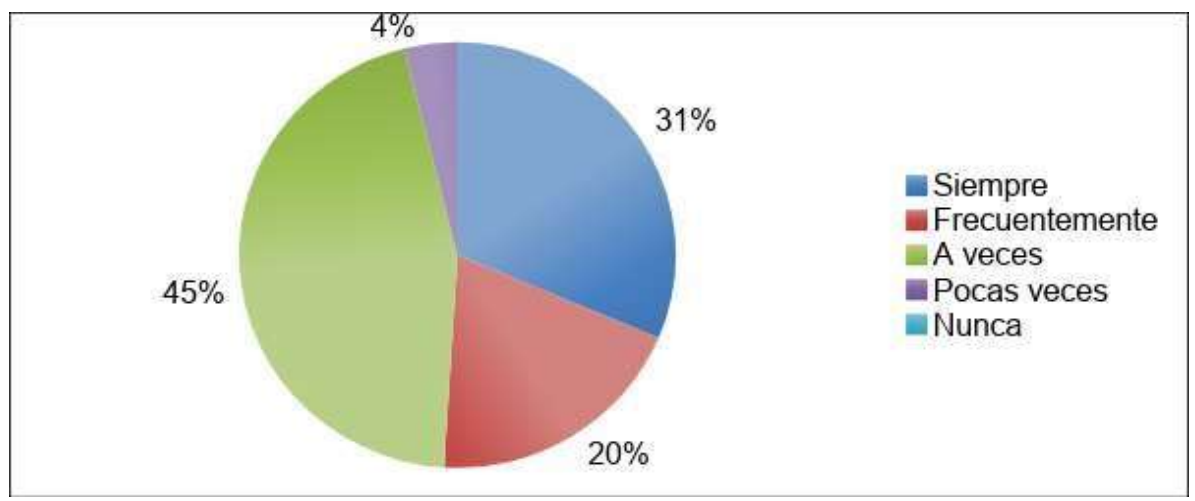

Gráfico 4. Uso de las TIC en el proceso de enseñanza y aprendizaje por su experiencia (16-30 años)

Fuente: Elaboración propia. 
En la actualidad, en los procesos de enseñanza y aprendizaje interviene alguno de los elementos de TIC que requiere conexión a internet y que puede utilizarse dentro o fuera del aula de clases. Para medir dicha característica, se consultó a los docentes acerca de las herramientas tecnológicas que emplea para comunicarse con los estudiantes con una pregunta multi-opción. El 55\% indica utilizar la Plataforma de la institución, el 16\% utiliza el Correo electrónico, el 2\% utiliza Facebook, el $4 \%$ utiliza Blog, el $18 \%$ utiliza Dispositivos móviles y el $5 \%$ utilizar otro tipo de herramienta.

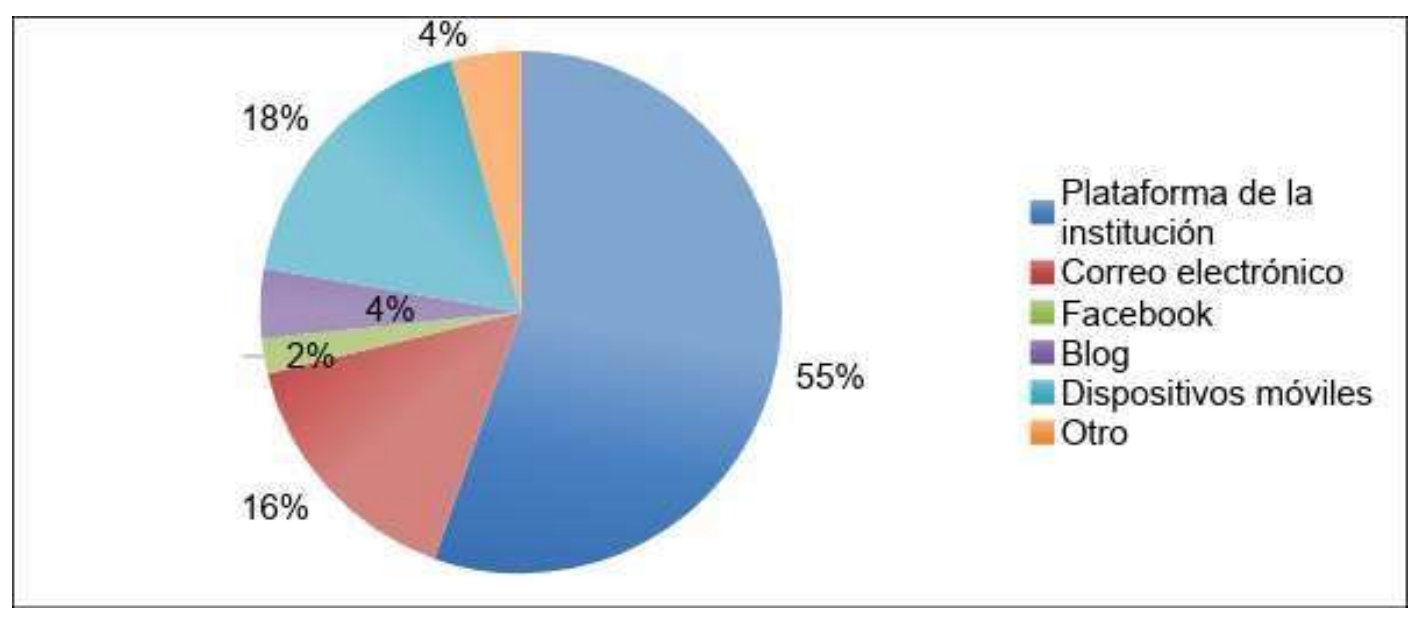

Gráfico 5. Herramientas que utilizan para comunicarse con los estudiantes Fuente: Elaboración propia.

La expectativa del uso de EVEA en el proceso de enseñanza y aprendizaje suele ser alta, por lo que se consideró consultar que impacto tiene actualmente en sus clases. De la totalidad de docentes de muestra, el 10\% considera un impacto Significativo, el $34 \%$ un impacto Medianamente significativo y el $57 \%$ un impacto Poco significativo. 


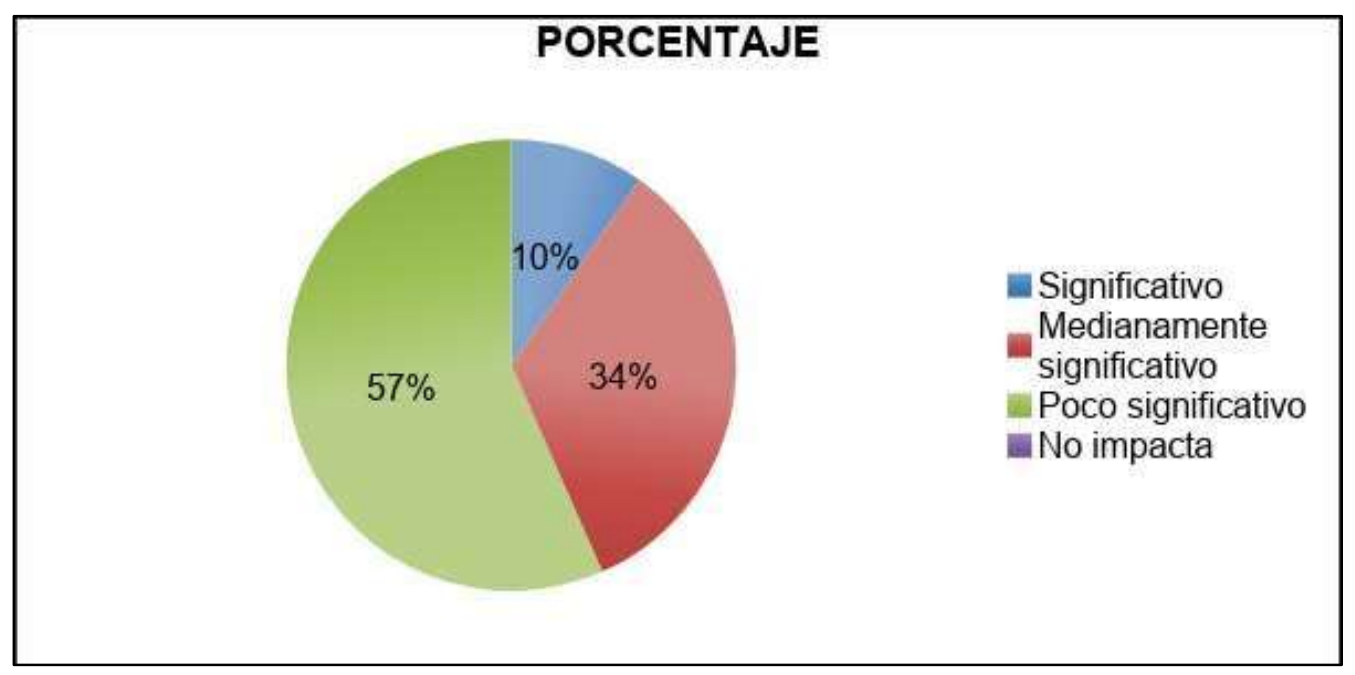

Gráfico 6. Impacto del uso de EVEA

Fuente: Elaboración propia.

\section{DISCUSIÓN}

Luego de haber realizado los respectivos análisis y de haber revisado en varios documentos sobre el uso de las TIC y la aplicación de lineamientos pedagógicos junto con el desempeño de los profesores usando no simplemente las TIC sino también los Entornos Virtuales de Aprendizaje, hemos visto cómo el actuar de los docentes ha cambiado con el paso de los años, más que todo por el impulso que el Gobierno de turno está realizando a nivel nacional.

Muchos docentes no están utilizando solo un presentador electrónico para dar sus clases, sino que crean Plataformas virtuales en webs gratuitas para poder interactuar e intercambiar información con los estudiantes, esto ha permitido que los alumnos puedan estar al día con el uso de tecnología. Los medios y recursos utilizados permiten mejorar el proceso enseñanzaaprendizaje y de esta manera, avanzar en el contenido y evaluación de las diversas materias que se dictan.

Consideramos que, con el paso de los años, el uso de los EVA y el desempeño de los docentes será cada vez mayor permitiendo mejorar la conectividad y la educación virtual. 
También se hace énfasis en el actuar de la nueva generación de docentes, quienes han nacido en la era tecnológica, lo cual permite que el uso de Entornos Virtuales sea más eficiente y la aplicación de recursos didácticos virtuales sirvan para fortalecer la enseñanza-aprendizaje y que de esta manera los alumnos tengan mejores herramientas para mejorar su parte cognitiva en las diversas áreas de estudio.

\section{CONCLUSIÓN}

Tomando en consideración el estudio realizado y enfocándonos en los resultados que se obtuvieron de las tres instituciones educativas se puede manifestar lo siguiente:

- Realizar capacitaciones en la creación y manejo de EVA, recalcando que el uso de TIC no se encuadra solamente en la utilización de un Presentador Electrónico.

- Fomentar en los docentes el manejo adecuado de los recursos que se pueden utilizar en los Entornos Virtuales, concientizando que el proceso de enseñanza-aprendizaje se debe fortalecer mas no dejar a un lado por el uso de las TIC.

- Motivar a los docentes que son de una edad avanzada, para el uso de recursos virtuales y de TIC con el propósito de mejorar el proceso enseñanza-aprendizaje.

\section{AGRADECIMIENTOS}

A las tres instituciones educativas de la ciudad de Guayaquil: Unidad Educativa Steiner Internacional, Unidad Educativa Ecomundo Babahoyo y Unidad Educativa Santa Luisa de Marillac, por la apertura de sus directivos para realizar las encuestas, entrevistas y el estudio de campo para obtener resultados fiables en este proceso investigativo. 


\section{REFERENCIAS BIBLIOGRÁFICAS}

Bustos S., A., \& Coll S., C. (2010). Los entornos virtuales como espacios de enseñanza y aprendizaje. Una perspectiva psicoeducativa para su caracterización y análisis. Revista mexicana de investigación.

educativa, $\quad 15(44), \quad$ 163-184. $\quad$ Recuperado de http://www.scielo.org.mx/scielo.php?script=sci arttext\&pid=S1405$\underline{66662010000100009 \& \operatorname{lng}=e s \& t \operatorname{lng}=e s .}$

Colorado, B. L., \& Edel-Navarro, R. (2012). La usabilidad de TIC en la práctica educativa. REDRevista de Educación a Distancia, (30).

Conde, M. Á., García, F. J. \& Humanante, P. R. (2013). Entornos Personales de Aprendizaje y Aulas Virtuales: una Experiencia con Estudiantes Universitarios. VAEP-RITA, 1 (4), 211-217.

Gómez, D. U., Pérez, D. J., \& Tamayo, L. P. (2007). Entornos virtuales de enseñanza aprendizaje. Universidad Territorial de las Tunas, 8 (78).

Guaña - Moya, E. J., Llumiquinga-Quispe, S. d., \& Ortiz-Remache, K. J. (2015). Caracterización de entornos virtuales de enseñanza aprendizaje (EVEA) en la educación virtual. Centro de Información y Gestión Tecnológica de Santiago de Cuba, 3 (17. Obtenido de http://www.redalyc.org/pdf/1815/181542152006.pdf

INEC (2012). Encuesta Nacional de Empleo, Desempleo y Subempleo (ENEMDU DIC- 2011).

Ledo, M. M., Hernández, M. G., Cao, M. N., \& Vidal, M. M. (2015). Entornos personales de $\begin{array}{llllll}\text { aprendizaje. } & \text { Scielo, } & 2 & -(14) . & \text { Obtenido de }\end{array}$ http://scielo.sld.cu/scielo.php?script=sci_arttext\&pid=S086421412015000400023 
Mestre, U., Valdés, P. R. \& Fonseca, J. J. (2007). Entornos virtuales de enseñanza aprendizaje. Ciudad de Las Tunas: Editorial Universitaria.

Ministerio de Educación (2019). Lineamientos Pedagógicos para el uso de recursos educativos digitales abiertos en el proceso de enseñanza aprendizaje. QuitoEcuador, Primera edición.

Rallo, R., Gisbert, M., Adell, J. \& Anaya, L. (1997). Entornos de formación presencial virtual ya distancia. 